\title{
Shock Tube for analysis of combustion of biofuels
}

\author{
Claudio Marcio Santana ${ }^{1}$, Jose Eduardo Mautone Barros ${ }^{2}$ \\ Universidade Federal de Minas Gerais ${ }^{1.2}$
}

claudiowsantana@gmail.com, mautone@demec.ufmg.br,

\begin{abstract}
A burning process in a combustion chamber of an internal combustion engine is very important to know the maximum temperature of the gases, the speed of combustion, and the ignition delay time of fuel and air mixture exact moment at which ignition will occur. The automobilist industry has invested considerable amounts of resources in numerical modeling and simulations in order to obtain relevant information about the processes in the combustion chamber and then extract the maximum engine performance control the emission of pollutants and formulate new fuels. This study aimed to general construction and instrumentation of a shock tube for measuring shock wave. As specific objective was determined reaction rate and ignition delay time of ethanol doped with different levels of additive enhancer cetane number. The results are compared with the delays measured for the ignition diesel and biodiesel.
\end{abstract}

\section{INTRODUCTION}

A shock tube is a metal tube in which a gas at low pressure and a high pressure gas are separated by a diaphragm. This diaphragm when it breaks in predetermined conditions produces shock waves moving the high pressure chamber known as a compression chamber or driver chamber to the low pressure chamber known as expansion or Driven. Were obtained in testing the ignition delay times Ethanol additive, diesel and biodiesel, and also measured the propagation velocity of the shock wave direct and reflected. 


\section{LITERATURE REVIEW}

A shock tube is a device used to create the gas flow in order to simulate conditions which are difficult to achieve in other test devices and ascertain the effects of thermal and dynamic fluid flow that, for example, the initial effects of an explosion. They are also used for studying aerodynamic flows under varying conditions of temperature and pressure, compressibility effects and conditions of combustion gases. In the simplest configuration one shock tube is a tube of great length with constant cross-sectional area, formed by two closed sections and separated by a diaphragm [1]. Figure 1 shows the design of a simple shock tube

Figure 1 - Simple Shock Tube.

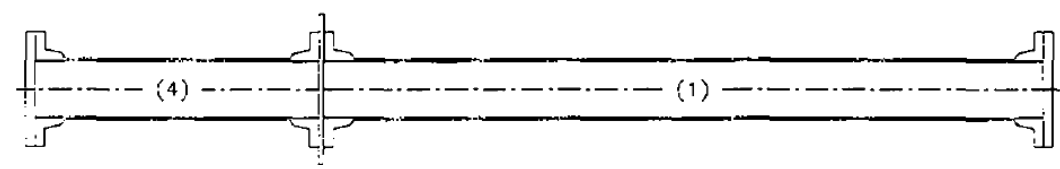

Source: Antonio (1997).

First of these sections called Driver section is pre-filled with a gas or gas mixture until a certain pressure, while another section called Driven remains at a pressure lower than the Driver section. When the pressure difference between the two sections is sufficient makes the diaphragm ruptures and a shock wave compression is driven toward the low pressure section (Driven). Instantly a wave of expansion propagates toward the high pressure section (driver). The gases in the sections may or may not be from the same species as may or may not be at different temperatures before rupture of the diaphragm. After the rupture of the diaphragm compression shock wave causes movement of the mass of gas increasing the temperature and pressure in the section Driven. The wave of expansion decreases the temperature and pressure of the gas as it moves to the Driver section [3]. Figure 2 shows the sections of high and low pressure shock tube before the rupture of the diaphragm.

Figure 2 - Representation of the sections of the shock tube before rupture of the diaphragm.

\begin{tabular}{|c|c|}
\hline $\begin{array}{c}4 \\
\text { High Pressure }\end{array}$ & $\begin{array}{c}1 \\
\text { LowPressure }\end{array}$ \\
\hline Driver Section & Diaphragm \\
\hline
\end{tabular}


Immediately after rupture of the diaphragm form a region called the contact surface on which the gas sections are Driven and driver and begin to mix with the movement of the gas mass inside the tube that region disappears [3]. Figure 3 shows the regions formed between the contact surfaces.

Figure 3 - Formation of the contact surface immediately after rupture of the diaphragm.

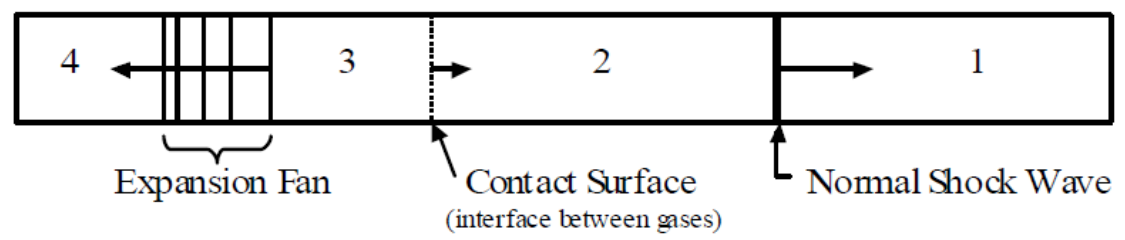

Source: McMillan (2004).

The compression wave (also called an incident) propagates to the right end of the tube; this movement causes an increase in temperature and pressure of the gas in the section Driven. Upon reaching the closed end of the tube the shock wave reflected and propagates toward the other end of the tube. In the movement of the reflected wave is back with the incident wave, the superposition of shock waves further increases the temperature and pressure of the gas in the Driven section. Therefore the reflected wave is responsible for causing often the dissociation and ionization of air. Also, the expansion wave moves toward the other end of the tube. When you reach the end, also closed, the wave is reflected and propagates back to the center of the tube. This process continues until the gas pressure and temperature to stabilize, which typically lasts less than one second [3].

\subsection{Analytical solution Tube Shock}

There are two parameters that determine the strength of the shock: the pressure ratio between the sections and Diver Driven (P4/P1) ratio and speed of propagation of sound in the respective sections Driver and Driven (a4/a1). The ratio of the speed of sound is determined by the ratio of specific heats and molecular weights of the gases used in sections Driver and Driven [3]. Figure 4 represents the conditions of shock tube before rupture of the diagram, assuming that the two sections are at the same temperature. 
Figure 4 - Condition shock tube before rupture of the diaphragm.

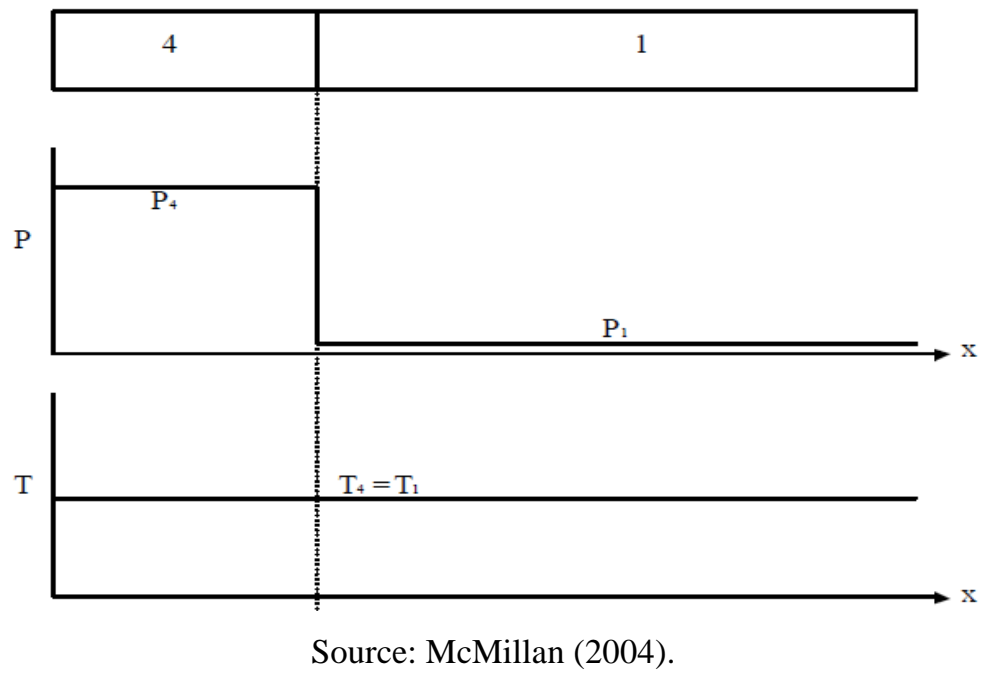

The principles of normal movement of the shock are used to develop the relationships between the regions 1 and 2 on each side of the compression wave and between regions 3 and 4 each side of the wave expansion. Although initially there are only two gases in the tube after the rupture of the diaphragm, there are four states gas temperature, pressure, density and specific heat well defined for each region. The front of the shock wave normal to the pressure wave of expansion, density, and temperature are the same initial conditions sections of low and high pressure respectively, that is, these regions are not affected by shock and expansion waves. Behind the normal shock wave is increased in pressure, density and temperature, while expanding wave behind these variables decrease. The region bounded by the shock wave and expansion wave is known as the contact surface [3]. Figure 5 shows the conditions of shock tube after rupture of the diaphragm.

Figure 5 - Conditions Tube Shock after diaphragm rupture.
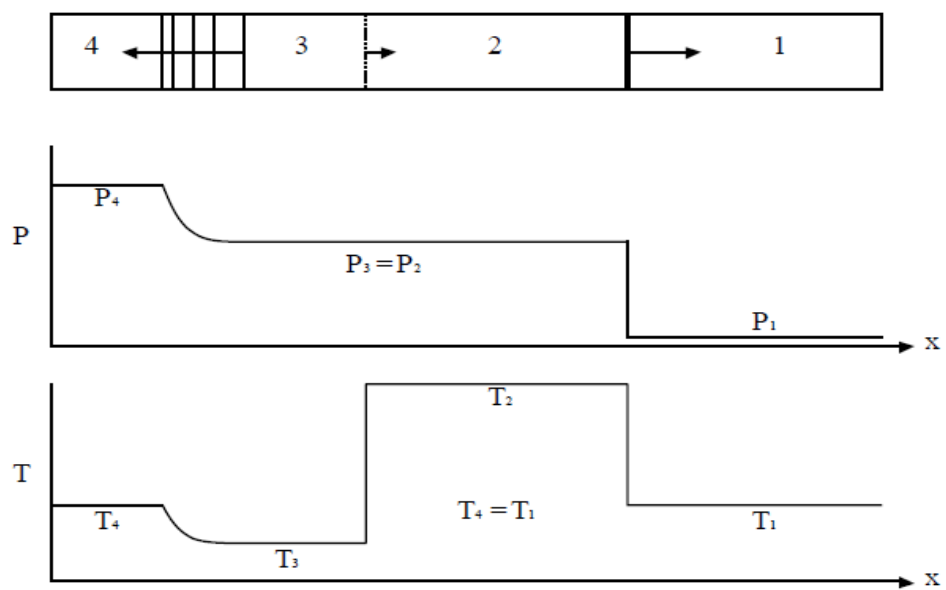

Source: McMillan (2004). 
The reflected shock wave and travels at a speed higher pressure than the incident wave [3]. Figure 6 shows the conditions of shock tube after reflection of the shock wave.

Figure 6 shows the conditions of shock tube after reflection of the shock wave.

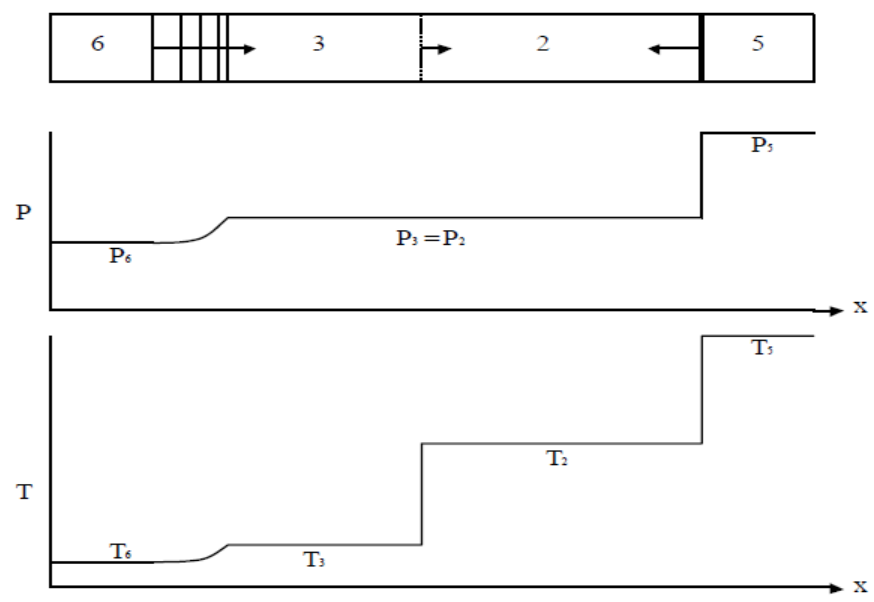

Source: McMillan (2004).

The speed of sound for each state gas must be calculated using the equation1:

$$
a=\sqrt{\gamma R T}
$$

$\gamma$ which is the ratio of specific heats of the gas, $\mathrm{R}$ is the universal gas constant and $\mathrm{T}$ is the gas temperature in the respective regions of the tube [3].

The Mach number can be determined using the following equation 2 [3].

$$
\frac{P_{4}}{P_{1}}=\frac{\gamma_{1-1}}{\gamma_{1+1}}\left[\frac{2 \gamma_{1}}{\gamma_{1-1}} M_{s}^{2}-1\right]\left[1-\frac{\frac{\gamma_{4-1}}{\gamma_{4+1}}\left(\frac{a_{1}}{a_{4}}\right)\left(M_{s}^{2}-1\right)}{M_{S}}\right]^{-\frac{2 \gamma_{4}}{\gamma_{4-1}}}
$$

Where Ms is the Mach number of the shock wave, the subscript 1 denotes the properties of the Driven section and the subscript 4 denotes the properties of the Driver section [3].

With the Mach number known to the pressure ratio on both sides of the shock wave (P2/P1) can be calculated using the ratio of normal shock given for equation3 [3]. 


$$
\frac{P_{2}}{P_{1}}=1+\frac{2 \gamma_{1}}{\gamma_{1}+1}\left(M_{s}^{2}-1\right)
$$

The pressure ratio $(\mathrm{P} 2 / \mathrm{P} 1)$ may be used to determine the temperature ratio $(\mathrm{T} 2 / \mathrm{T} 1)$ on both sides of the shock wave given for equation 4 [3].

$$
\frac{T_{2}}{T_{1}}=\frac{P_{2}}{P_{1}}\left(\frac{\frac{\gamma_{1}+1}{\gamma_{1}-1}+\frac{P_{2}}{P_{1}}}{1+\frac{\gamma_{1}+1}{\gamma_{1}-1} \frac{P_{2}}{P_{1}}}\right)
$$

The temperature of the gas behind the shock wave can be used to predict the dissociation and ionization of the air. The relationship between gas pressure and shock Mach number is asymptotic. Therefore, for each pair of gases Mach number there is a theoretical can be achieved. This theoretical maximum number indicated by a star subscribed can be calculated by equation 5 [3].

$$
M_{s}^{*}=\frac{\gamma_{1}+1}{2\left(\gamma_{4}-1\right)} \frac{a_{1}}{a_{4}}+\sqrt{\left[\frac{\gamma_{1}+1}{2\left(\gamma_{4}-1\right)} \frac{a_{1}}{a_{4}}\right]^{2}+1}
$$

The velocity of the shock wave reflected depends upon the velocity of the incident shock wave and can be determined by the following equation 6 [3].

$$
\frac{M_{R}}{M_{R}^{2}-1}=\frac{M_{S}}{M_{S}^{2}-1} \sqrt{1+\frac{2\left(\gamma_{1}-1\right)}{\left(\gamma_{1}+1\right)^{2}}\left(M_{S}^{2}-1\right)\left(\gamma_{1}+\frac{1}{M_{S}^{2}}\right)}
$$

The increased pressure of the shock wave reflected depends on the speed of the incident shock wave; this ratio can be calculated by the equation 7 [3].

$$
\frac{P_{5}}{P_{2}}=1+\frac{2 \gamma_{1}}{\gamma_{1}+1}\left(M_{R}^{2}-1\right)
$$

The shockwave reflected stops the motion of the mass of gas, so that the propagation velocities of the gases behind the shock wave and the reflected shock wave front incident tend to zero. The following equation shows the relationship of movement of these gases [3].

$$
\frac{2 a_{1}}{\gamma_{1}+1}\left(M_{S}-\frac{1}{M_{s}}\right)=\frac{2 a_{2}}{\gamma_{1}+1}\left(M_{R}-\frac{1}{M_{R}}\right)
$$


The calculations involving speed of the gas molecules are simple and can be determined by the following equation [3].

$$
\operatorname{Mach} M=\frac{V}{a}
$$

The calculation of the actual speed of the wave must take into account the mass movement of the gas behind the shock wave incident. Thus the rate can be calculated by the following equation [3].

$$
V_{R}=M_{R} a_{2}-V_{2}
$$

To determine the relationship between pressure waves reflected and incident shock using only the incident wave data, the following equation can be used [3].

$$
\frac{P_{5}}{P_{2}}=\left(\frac{\frac{\gamma_{1}+1}{\gamma_{1}-1}+2-\frac{P_{1}}{P_{2}}}{1+\frac{\gamma_{1}+1}{\gamma_{1}-1} \frac{P_{1}}{P_{2}}}\right)
$$

With the known compression ratio, the temperature ratio can be determined by equation 12 [3].

$$
\frac{T_{5}}{T_{2}}=\frac{P_{5}}{P_{2}}\left(\frac{\frac{\gamma_{1}+1}{\gamma_{1}-1}+\frac{P_{5}}{P_{2}}}{1+\frac{\gamma_{1}+1}{\gamma_{1}-1} \frac{1}{P_{2}}}\right)
$$

The temperature and pressure behind the reflected shock wave can be calculated knowing only the Mach number of the incident shock wave. This value can be determined from the velocity of the gas driven and wave velocity [3].

\section{METHODOLOGY}

The methodology involves the construction of the instrumentation tube as well as shock and combustion tests without the shock tube. 
Was developed by the laboratory combustion of the Federal University of Minas Gerais (UFMG) one tube to withstand shock pressures of 3 Mega Pascal and possible peaks of 13.7 Mega Pascal. This tube was made of stainless steel, with total length of 7.00 meters divided into 7 modules with 1.00 meters each, inner diameter of $97.18 \mathrm{~mm}$ and thickness of $8.56 \mathrm{~mm}$ sidewalls. One of the modules has six windows for optics and instrumentation holes 14 for installing pressure sensors and temperature. As for the other six modules only have 4 holes for installation of sensors. These holes can also be used for installation of fuel injector valves and spark plugs. Figure 7 shows the schematic of the shock tube.

Figure 7 - Schematic of the shock tube.

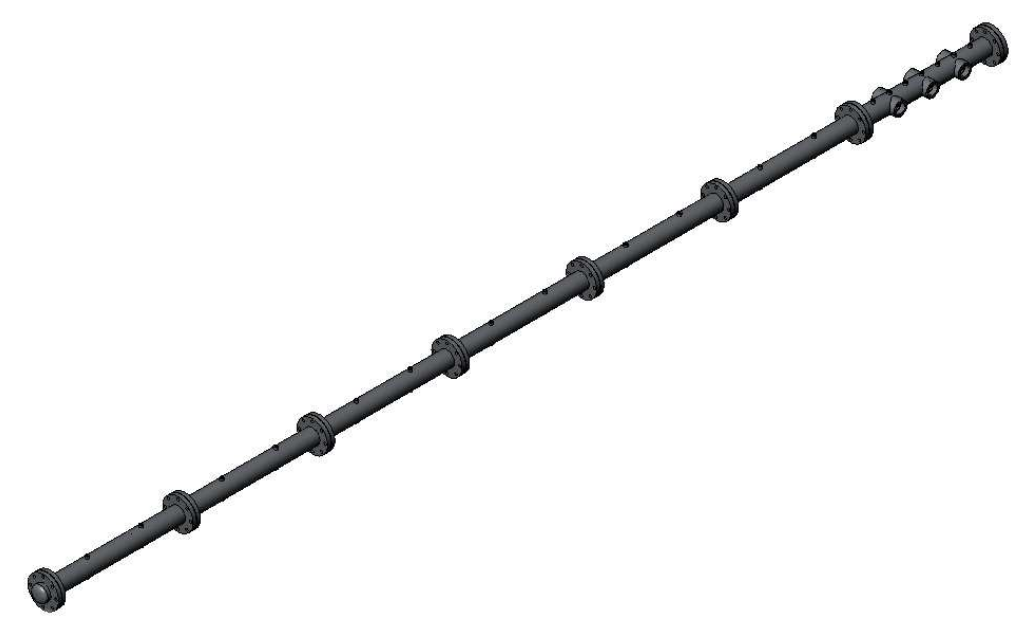

Source: Combustion Laboratory, UFMG (2010).

\section{RESULTS AND DISCUSSION}

\subsection{Test tube in Shock without combustion}

Following are the results and analysis of tests conducted without combustion in tube shock. Four experiments were conducted with the tube ends closed and a test with one end open. In all tests, we used a data acquisition rate of $600 \mathrm{~Hz}$ (6000 points in 10 seconds).

The first test was performed with both ends of the shock tube closed in this assay four membranes used were aluminum with a thickness of $0.03 \mathrm{~mm}$ each. Four membranes used in order to increase the burst pressure, as in earlier tests with a single membrane were observed one burst pressure of approximately 4 Bar. 
The membranes were placed between the sections Driver (high pressure region) and Driven (low pressure region), this region separation of the sections is located in the middle of the tube, and i.e. each section is three meters long. Figure 8 shows the location of the membrane between the driver sections Driven left and right.

Figure 8 shows the location of the membrane between the driver sections Driven left and right.

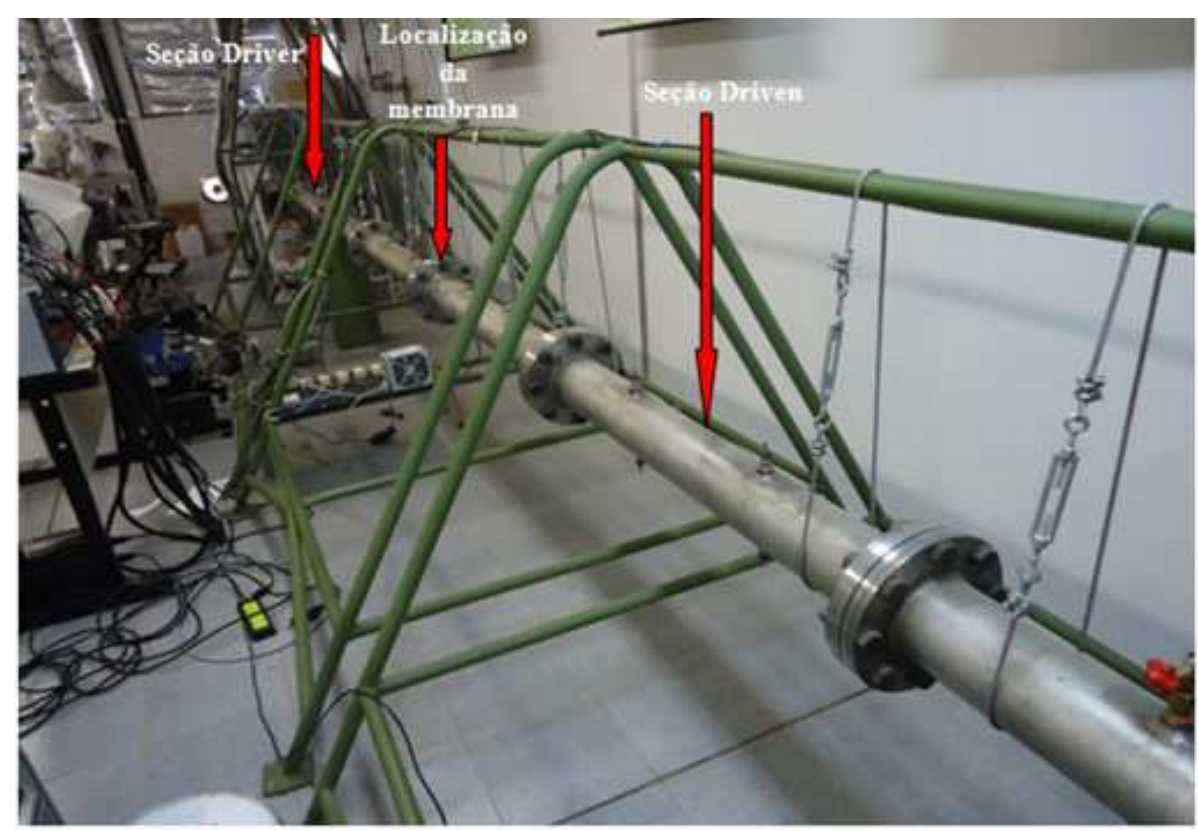

Source: Combustion Laboratory, UFMG (2010).

Compressed air from the internal laboratory was injected in the Driver section. The pressure was monitored by a pressure gauge and three pressure sensors installed along the tube. Figure 9 shows a 10 second acquisition of the three sensors on the pipe during the test membrane disruption. 
Figure 9 - Test of membrane disruption within 10 seconds.

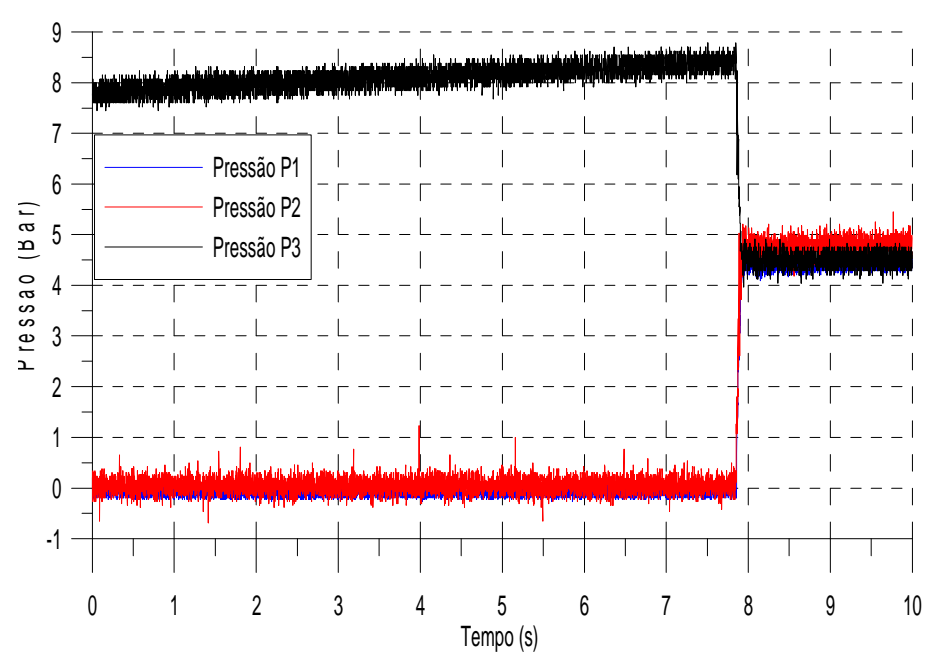

Source: Combustion Laboratory, UFMG (2012).

The figure above shows an increase of pressure in the Driver section (pressure P3), this increase refers to the injection of compressed air in the section. Pressure Driven section (pressures P1 and P2) does not change, because the sections are insulated by the membranes of aluminum. The figure also shows the moment of rupture of the membrane which is characterized by the fallen $\mathrm{P} 3$ and the pressure was recorded on pressure increase at P1 and P2. Figure 10 shows in a range of 1 second moment of rupture of the membrane.

Figure 10 - Test of membrane disruption within 1 second.

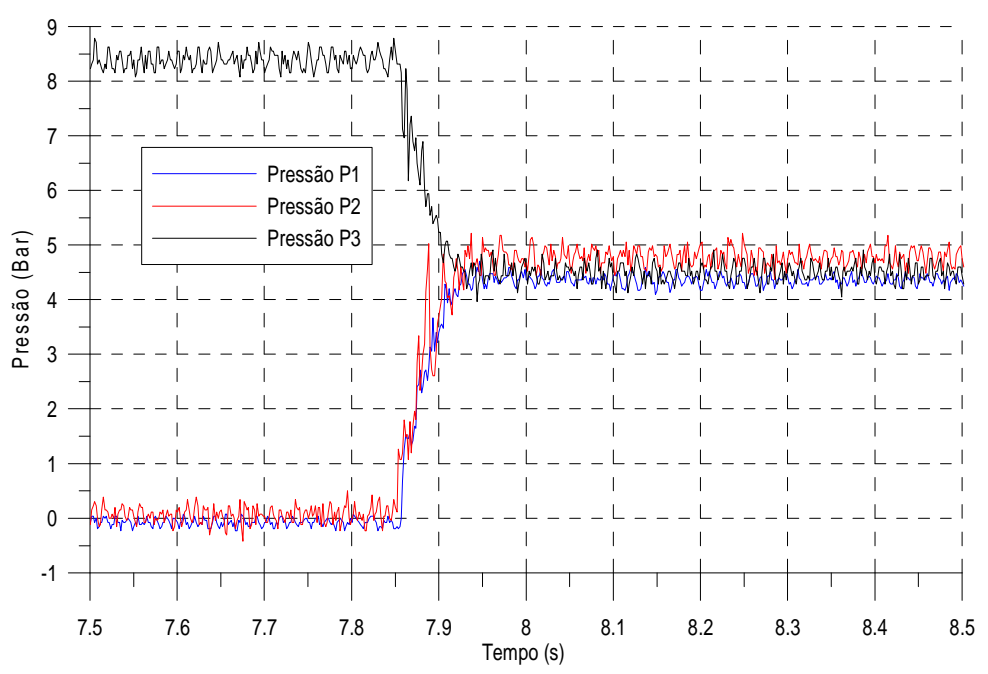

Source: Combustion Laboratory, UFMG (2012).

The figure shows that as the rupture of the membrane pressure in the Driver section and pressure drops in the Driven section increases. It may be observed from the figure that the sensor P2 feel 
the shock sensor P1 before, this fact was expected, since the sensors P1 and P2 are located 700 to $2700 \mathrm{~mm}$ from the membrane, respectively, so the shock waves produced on the basis of membrane disruption reach before P1 P2 sensor.

The propagation velocity of the shock wave Driven section was calculated using the equation 1 . According to this equation for an air temperature of $300 \mathrm{~K}$ in the tube, the velocity of propagation of the shock wave will be $345,3 \mathrm{~m} / \mathrm{s}$, that is, the wave travels a distance of 1 meter in 3 seconds or milliseconds 0.003 . For more precise visualization of the shock wave propagation time to membrane disruption was reduced to 0.2 second divided in intervals of 10 milliseconds, as shown in Figure 11.

Figure 11 - Test of membrane disruption within 0,2 seconds.

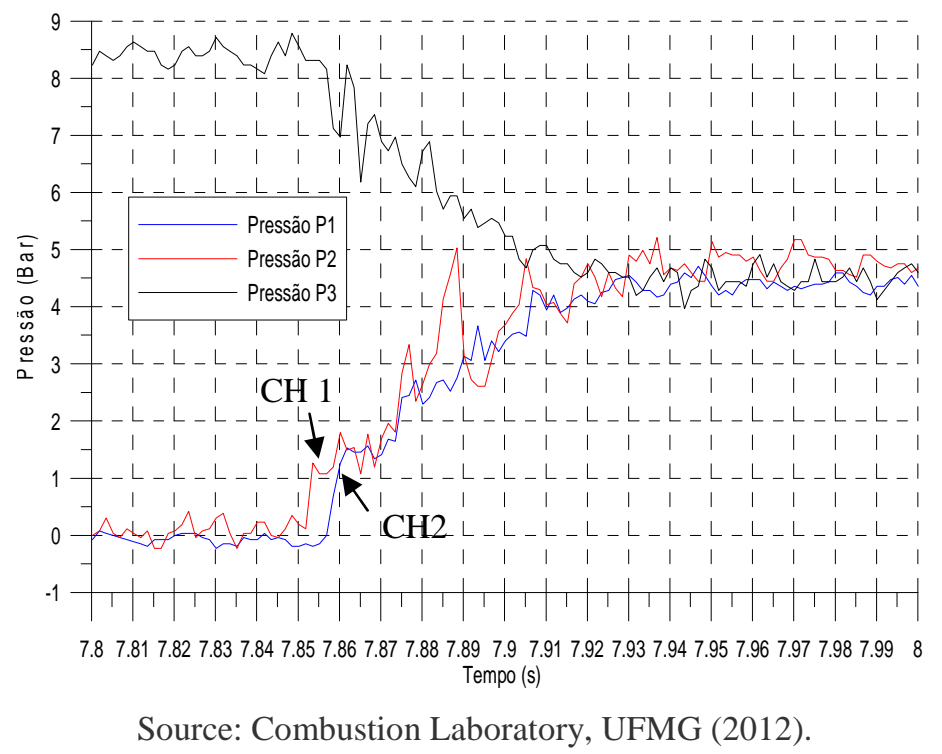

The figure above shows the point $\mathrm{CH} 1$ representing the time at which the sensor P2 (located 700 $\mathrm{mm}$ from the membrane) feels the shock due to the first passage of the shock wave. The $\mathrm{CH} 2$ also shows the point when the sensor P1 (located at 2700 and $2000 \mathrm{~mm}$, respectively, of the sensor membrane and P2) senses the perturbation due to the passage of the wave. In the graph realize that the two points are distant $\mathrm{CH} 1$ and $\mathrm{CH} 2$ of about 6 milliseconds, consistent with this analysis that the distance between the location of pressure sensors P1 and P2 (2 meters) and the speed of propagation of the wave, which travels a distance of 1 meter in 3 milliseconds.

Upon reaching the closed end of the tube the shock wave reflected and propagates toward the other end of the tube. In moving back the reflected wave to incident wave overlaps increasing 
even further increases the temperature and pressure of the gas in the section Driven. In the chart above was not possible to detect the time of passage of the wave reflected by the sensors P1 and P2. In this case as the sensor P1 is located closer to the closed end, it should feel the wave propagation before the sensor $\mathrm{P} 2$. The failure to observe the times of passage of the wave by the sensors can be related to the pressure ratio between sections driver and driven, that in this assay was $8: 1$.

The second test was also conducted with the tube ends closed, but the membrane used in the assay was of copper with thickness of 0.1 millimeters. For this test outer cylinder was needed to assist in the filling tube, since the network is limited in the laboratory 8 Bar pressure. Figure 12 shows in a range of 10 seconds time to membrane disruption.

Figure 12 - Test of membrane disruption within 10 seconds

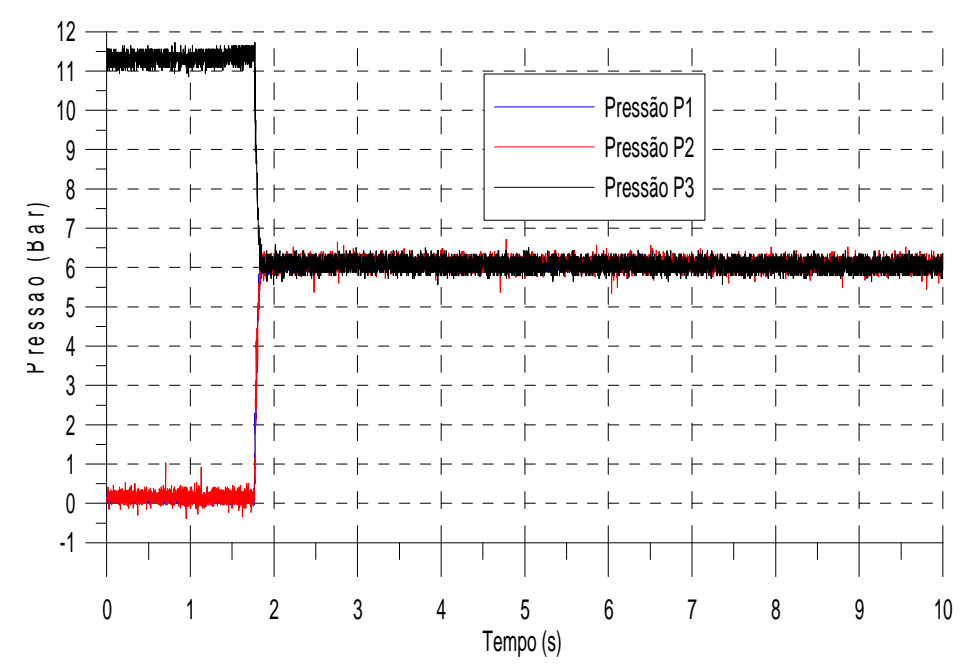

Source: Combustion Laboratory, UFMG (2012).

The figure above realizes the burst pressure of the membrane was approximately 12 Bar. Figure 13 shows in a range of 1 second moment of rupture of the membrane. 
Figure 13 - Test of membrane disruption within 1 second.

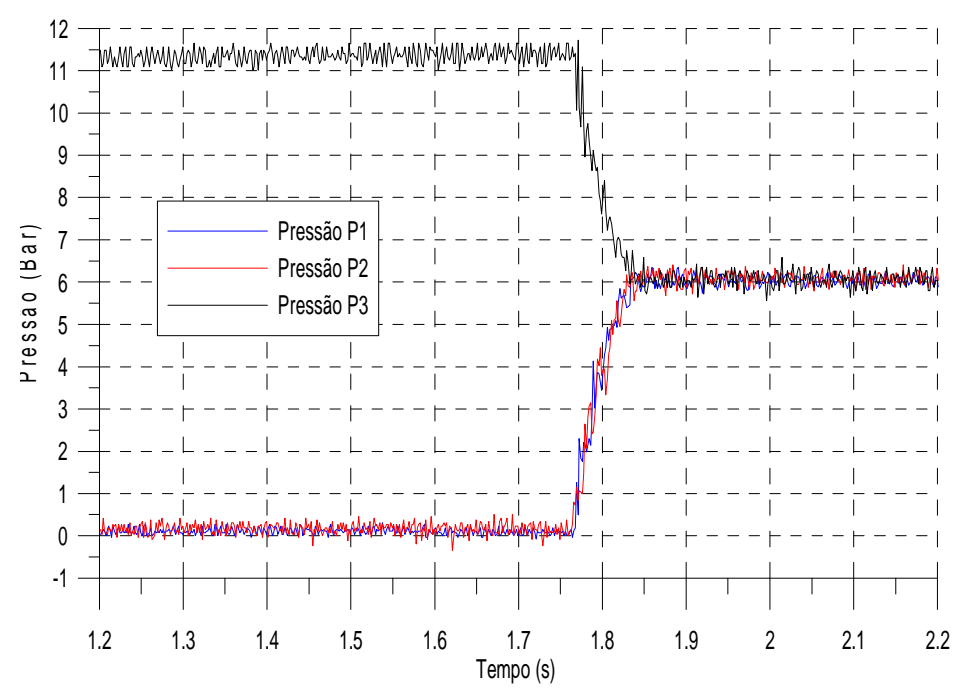

Source: Combustion Laboratory, UFMG (2012).

Figure 14 shows in a range of 0.2 second moment of rupture of the membrane.

Figure 14 - Test of membrane disruption in the range of 0.2 seconds.

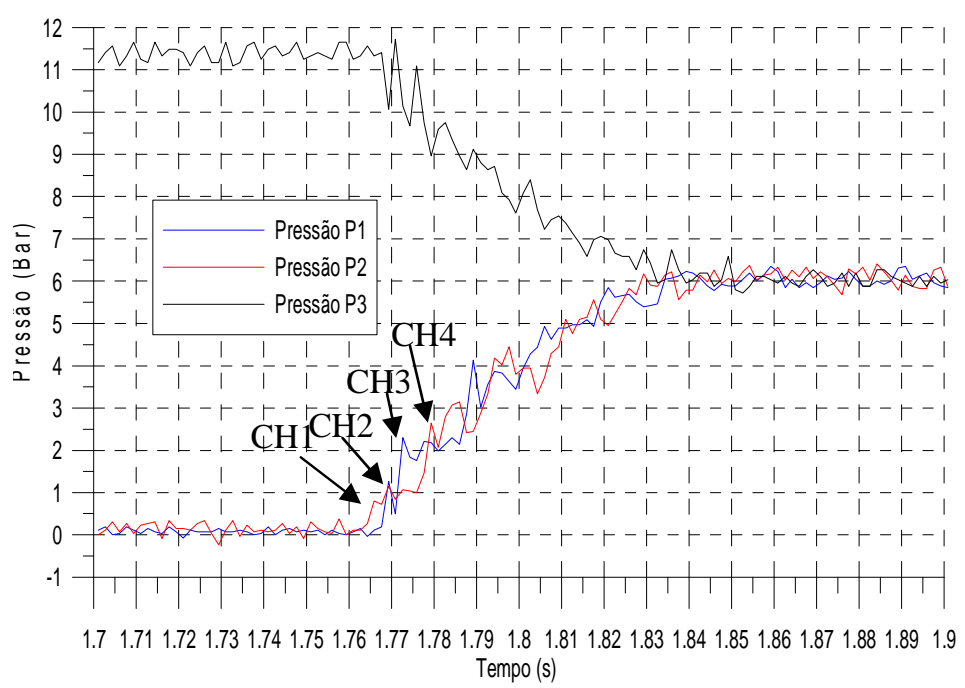

Source: Combustion Laboratory, UFMG (2012).

It may be observed in the test with the first similarity with respect to the incident shock wave, that is, the points marked on the graph $\mathrm{CH} 1$ and $\mathrm{CH} 2$ respectively represent the moments where P1 and P2 sensors sense the passage of the shock wave (first the sensor P2, and after about six milliseconds sensor P1). In this assay it was possible to identify the moment in which the sensor sensed the shock wave reflected. Analyzing the graph realize that the sensor senses the passage $\mathrm{P} 1$ of the reflected wave (dot $\mathrm{CH} 3$ ) 3 feel milliseconds after the passage of the incident 
wave. The sensor P2 felt passage of the reflected wave after about 6 milliseconds P1 sensor sense the passage of the reflected wave. This analysis is in agreement with the distance of the sensor location and velocity of wave propagation.

The next test was also performed with the ends of the shock tube closed, however a membrane was used to cover 0.2 millimeters. The figure 15 shows in a 10 second interval time to membrane disruption.

Figure 15 - Test of membrane disruption within 10 seconds

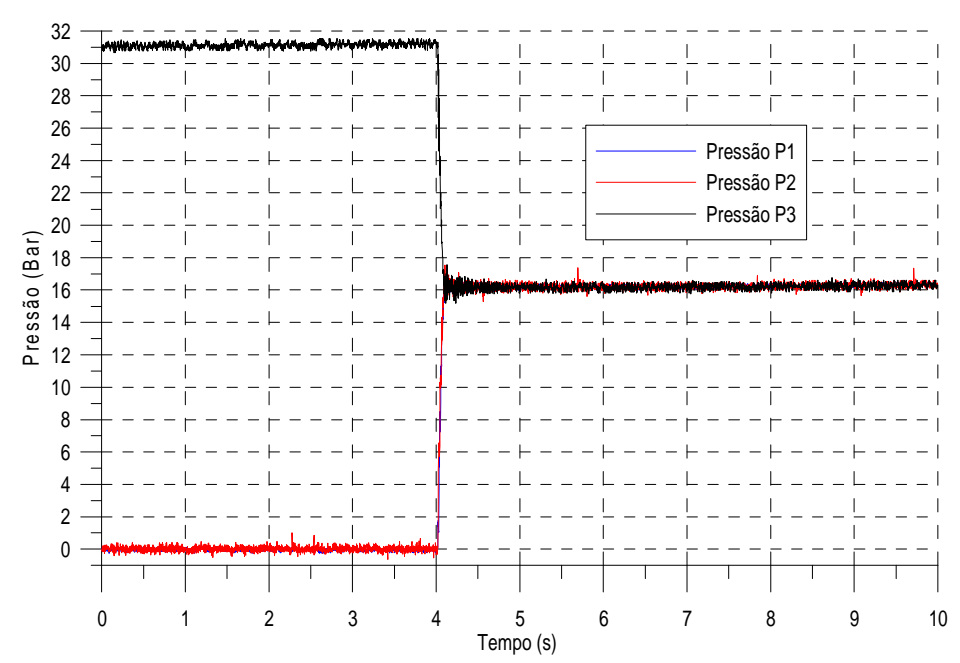

Source: Combustion Laboratory, UFMG (2012).

Verifies that with the increasing thickness of the membrane covering the burst pressure was approximately 31 bar. The figure 16 shows in a 1 second interval time to membrane disruption.

Figure 16 - Test of membrane disruption within 1 second.

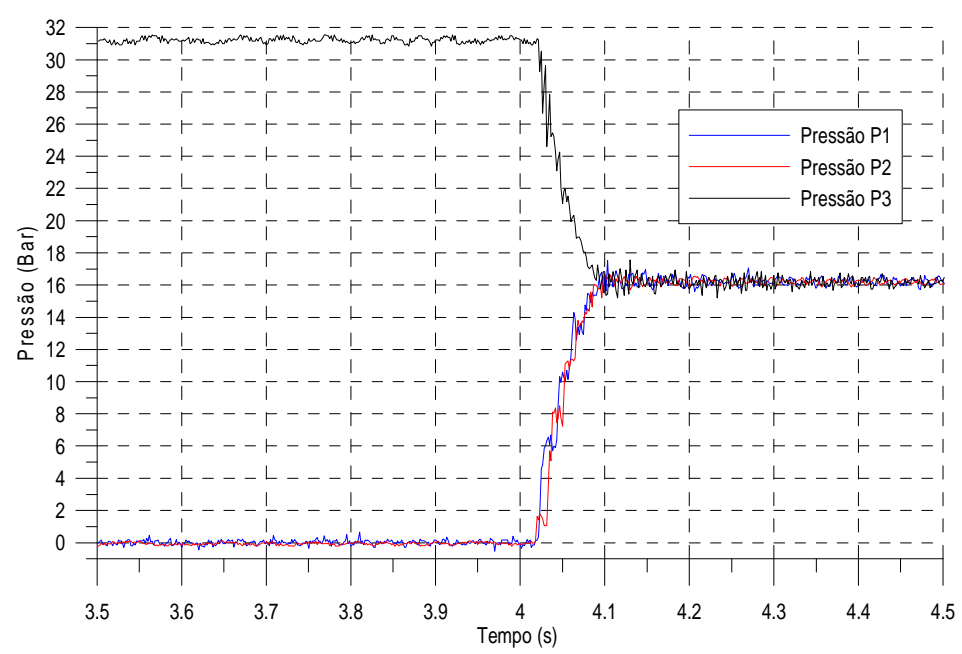

Source: Combustion Laboratory, UFMG (2012). 
The figure shows 17 at an interval of 0.2 second moment of rupture of the membrane.

Figure 17 - Test of membrane disruption in the range of 0.2 seconds.

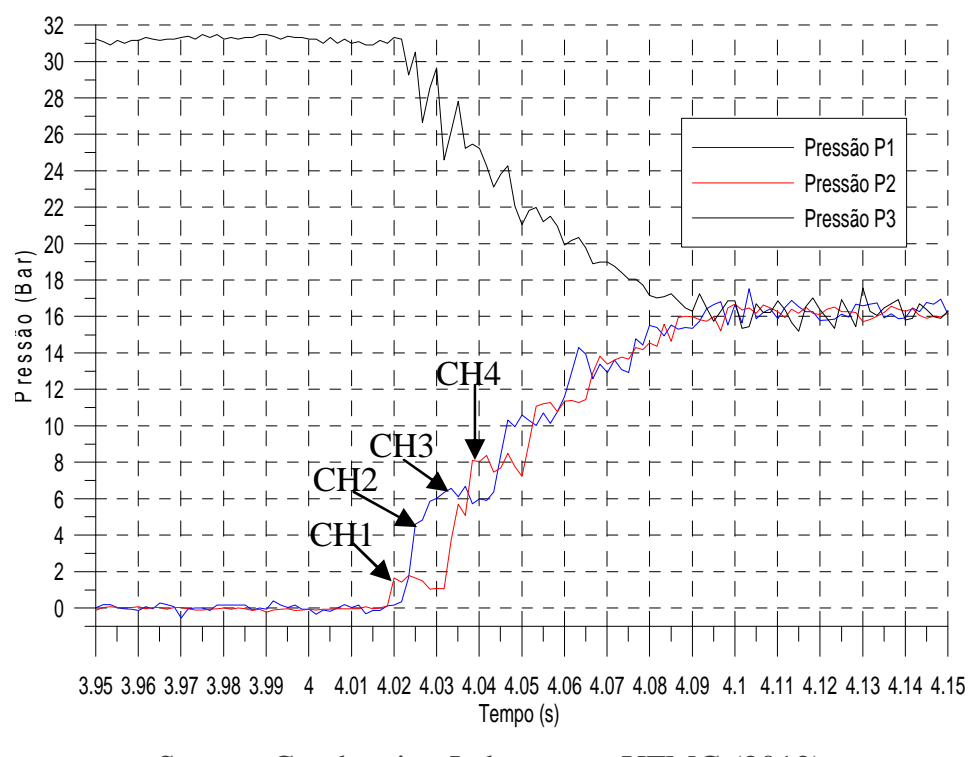

Source: Combustion Laboratory, UFMG (2012).

It may be observed in the test with the first similarity with respect to the incident shock wave, that is, the points marked on the graph $\mathrm{CH} 1$ and $\mathrm{CH} 2$ respectively represent the moments where P1 and P2 sensors sense the passage of the shock wave (first the sensor P2, and after about six milliseconds sensor P1). In this assay could also identify the time when the shock sensors felt reflected wave. Analyzing the graph realize that the sensor senses the passage P1 of the reflected wave (point $\mathrm{CH} 3$ ) 3 feel milliseconds after the passage of the incident wave. The sensor P2 felt passage of the reflected wave ( $\mathrm{CH} 4$ point) after about 6 milliseconds $\mathrm{P} 1$ sensor sense the passage of the reflected wave. This analysis is in agreement with the distance of the sensor location and velocity of wave propagation discussed above.

\section{CONCLUSIONS}

Tests on experimental shock tube without combustion was possible to determine the velocities of propagation of shock waves incident and reflected. In tests with combustion was to measure the ignition delay times of ethanol additive, diesel and biodiesel with different levels of additives. 


\section{REFERENCES}

[1] ANTONIO, M. C. N. Efeito do Pistão Gasoso em Tubo / Túnel de Choque Quando Operado na Condição de Equilíbrio de Interface. Tese apresentada a Divisão de Pós- Graduação do Instituto Tecnológico de Aeronáutica como parte dos requisitos para a obtenção do titulo de Doutor em Ciências no Curso de Engenharia Aeronáutica na área de Aeronáutica, propulsão e Energia, 1997.

[2] CANCINO, L. R. Autoignition of gasoline surrogate mixtures at intermediate temperatures and high pressures: Experimental and numerical approaches. Proceeding of the Combustion institute, 2009.

[3] MCMILLAN, R.J. Shock tube investigation of pressure and ion sensors used in pulse detonation engine research. Thesis of Degree of Master of Science in Aeronautical Engineering, 2004. 